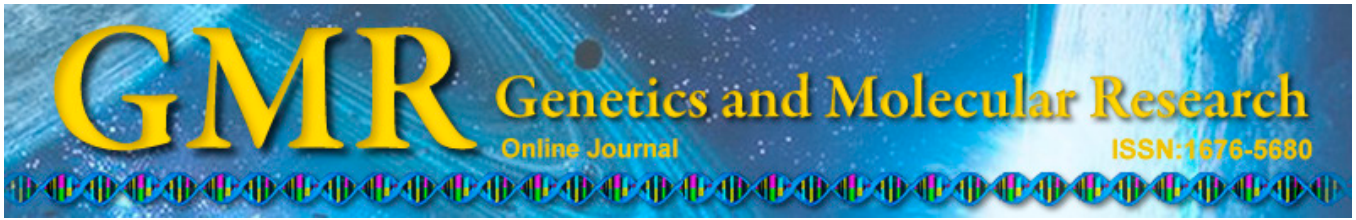

\title{
Role of salubrinal in protecting cardiomyocytes from doxorubicin-induced apoptosis
}

\author{
N. Gong ${ }^{1}$, J.H. Wu ${ }^{1}$, Z.S. Liang ${ }^{1}$, W.H. Jiang ${ }^{1}$ and X.W. Wang ${ }^{2}$ \\ ${ }^{1}$ Department of The Second General Surgery, \\ The Third Xiangya Hospital, Central South University, Hunan, Changsha, China \\ ${ }^{2}$ Department of Vascular Surgery, Xiangya Hospital, Central South University, \\ Hunan, Changsha, China \\ Corresponding author: X.W. Wang \\ E-mail: XianWeiWangxy@163.com
}

Genet. Mol. Res. 14 (4): 12377-12385 (2015)

Received April 17, 2015

Accepted July 14, 2015

Published October 16, 2015

DOI http://dx.doi.org/10.4238/2015.October.16.4

\begin{abstract}
We determined whether salubrinal can protect cardiomyocytes from doxorubicin-induced apoptosis and explored the related mechanisms to provide experimental evidence for exploring novel drug candidates to decrease cardiac toxicity. Neonatal rat cardiomyocytes were isolated, cultured in vitro, and pretreated with salubrinal $(10,20$, or $40 \mu \mathrm{M}$ ) to observe their response to doxorubicin-induced cell apoptosis. Lactate dehydrogenase assay, terminal deoxynucleotidyl transferase-mediated deoxyuridine triphosphate nick end-labeling staining, and flow cytometry were used to assess the extent of cardiomyocyte apoptosis. Fluorescent probes conjugated with 2',7'-dichlorofluorescein diacetate and a chemiluminescence assay were used to detect the production of reactive oxygen species. Western blotting was employed to quantify expression levels of cleaved caspase-3, cytosolic cytochrome c, and B-cell lymphoma-extra large (Bcl-xL). The mechanisms of salubrinal-related functions were also explored. Salubrinal effectively inhibited doxorubicin-induced reactive oxygen species production and
\end{abstract}


nicotinamide adenine dinucleotide phosphate oxidase activation, decreased the levels of cleaved caspase- 3 and cytosol cytochrome c, and increased Bcl-xL expression, thereby protecting cardiomyocytes from doxorubicin-induced apoptosis. Furthermore, salubrinal was found to protect cardiomyocytes by decreasing the dephosphorylation of eukaryotic translation initiation factor $2 \alpha(\mathrm{eIF} 2 \alpha)$. Salubrinal can protect cardiomyocytes from doxorubicin-induced apoptosis through its effects on eIF $2 \alpha$. It possibly ameliorates cardiac toxicity and can be used in clinical practice.

Key words: Salubrinal; Doxorubicin; Cardiac toxicity; Cell apoptosis; eIF $2 \alpha$

\section{INTRODUCTION}

With advances in diagnostic and treatment methods, the survival rate of cancer patients has increased significantly in recent years. Data show that $83 \%$ of children with newly diagnosed cancers survive more than 5 years (Silverman et al., 2010; Tarver, 2012). However, despite such tremendous progress in cancer treatment, medication side effects occur in many patients. For example, anthracycline, which is often used in treating hematological and solid tumors, has been reported to cause impairments in other organs in addition to effectively killing tumor cells (Lipshultz et al., 2008). A study showed that $74 \%$ of patients using anthracycline drugs developed chronic organ damage, and $43 \%$ of cancer survivors had more severe conditions (Oeffinger et al., 2006). The statistical data from some studies have led researchers to postulate cardiac toxicity as one of the most important causes of death or disability in cancer survivors (Lipshultz and Adams, 2010; Tukenova et al., 2010). Therefore, exploring the possible elimination of cardiac toxicity is necessary.

Studies have shown that endoplasmic reticulum-activated cell apoptosis plays an important role in the development of cardiac toxicity (Chua et al., 2006; Wang et al., 2012). Therefore, one possible approach to eliminating cardiac toxicity is through the regulation of endoplasmic reticulum-activated apoptosis. Salubrinal is a recently discovered selective inhibitor of the dephosphorylation of eukaryotic translation initiation factor $2 \alpha$ (eIF2 $\alpha$ ) (Boyce et al., 2005). It protects against endoplasmic reticulum-activated cell apoptosis or cardiomyocyte apoptosis (Dalal et al., 2012). Therefore, salubrinal could work as a new drug candidate for treating cardiac toxicity. Doxorubicin is a drug used in cancer chemotherapy. Its most serious side effect is life-threatening heart damage. However, the detailed mechanism by which doxorubicin induces cardiac toxicity has not been well established.

This study utilized an in vitro model of doxorubicin-induced cardiac toxicity using primary cultured rat cardiomyocytes to evaluate the protective effect of salubrinal on cardiomyocytes. We also elucidated the possible mechanisms involved by assaying the level of phosphorylation in eIF2 $\alpha$.

\section{MATERIAL AND METHODS}

\section{Primary culture of rat cardiomyocytes}

Rats were used for all experiments, and all procedures were approved by the Animal 
Ethics Committee of The Third Xiangya Hospital of Central South University, Hunan, China. The primary culture of cardiomyocytes from neonatal rats followed the methods described previously (Chen et al., 2008). In brief, the ventricle of neonatal (postnatal day 1-2) Sprague Dawley rats was separated and cut into approximately $1-\mathrm{mm}^{3}$ pieces. The tissue samples were then digested with $0.25 \%$ type IV collagenase for $1 \mathrm{~h}$, spread evenly in complete medium (Dulbecco's modified Eagle's medium with $10 \%$ fetal bovine serum and streptomycin), and cultured in $5 \% \mathrm{CO}_{2}$ at $37^{\circ} \mathrm{C}$ in an incubator for 2-4 days. Adherent and fused cells were subcultured, collected, and frozen at $-80^{\circ} \mathrm{C}$ for future use.

\section{Drug treatment}

Cardiomyocytes collected using the above method were cultured in the complete medium also described above. They were then divided into different drug pretreatment groups: 1) 4-h treatment with different concentrations of salubrinal $(10,20$, and $40 \mu \mathrm{M})$; 2) 24-h incubation with $1 \mu \mathrm{M}$ doxorubicin; and 3) 24-h treatment with doxorubicin following 4-h culture with different concentrations of salubrinal $(10,20$, and $40 \mu \mathrm{M})$. A further control group was cultured without drugs. Assays were performed on all treated cells as described in the following sections.

\section{Terminal deoxynucleotidyl transferase (TdT)-mediated deoxyuridine triphosphate nick end-labeling (TUNEL) staining}

TUNEL staining was performed using a TUNEL assay kit (Roche, Basel, Switzerland) following the manufacturer instructions. Five fields in which cells with dark brown granular signals were defined as having positive signals were randomly selected using a microscope at high magnification. The rate of cell apoptosis was calculated using the formula:

Rate of cell apoptosis $=$ total number of positive cells in the five fields / total number of all cells in the five fields $\times 100 \%$.

\section{Lactate dehydrogenase assay}

A test kit (Jiancheng Corp., China) was used to determine the level of lactate dehydrogenase in the cultured cardiomyocytes following the manufacturer instructions.

\section{Assay of cytosolic reactive oxygen species, superoxides, and nicotinamide adenine dinucleotide phosphate (NADPH) oxidase activity}

The detection of reactive oxygen species followed the protocol described previously (Eruslanov and Kusmartsev, 2010). In brief, $30 \mu \mathrm{M}$ 2',7'-dichlorofluorescein diacetate fluorescent dye was added to the cells of the different drug pretreatment groups and incubated for $1 \mathrm{~h}$. Treated cells were then digested and washed, and fluorescence intensity was measured to quantify the level of reactive oxygen species. The numbers of cells in the different groups were also counted to calibrate the fluorescent signal strength. The superoxides assay followed the method reported elsewhere (Wung et al., 1997).

The measurement of NADPH oxidase activity also followed a previously reported method (Juan et al., 2007). In brief, the microbodies in the cardiomyocytes were separated using an ultracentrifuge and dissolved in $150 \mu \mathrm{L}$ reaction buffer $(50 \mathrm{mM}$ potassium phosphate 
buffer, $\mathrm{pH} 7$, containing $1 \mathrm{mM}$ ethylene glycol tetraacetic acid, $150 \mathrm{mM}$ sucrose, $5 \mu \mathrm{M}$ lucigenin, and $100 \mathrm{mM} \mathrm{NADPH}$ ). The fluorescence intensity was measured using an Orion microplate reader every $60 \mathrm{~s}$. The protein concentration was also quantified using a BCA Protein Assay Kit (Pierce Corp., USA). The activity of NADPH oxidase was calculated as the ratio of the relative fluorescence intensity to the protein concentration.

\section{Western blot analysis of protein expression}

Cardiomyocytes were lysed by cell lysis buffer (Biyuntian Corp., China) and centrifuged at 12,000 rpm for $10 \mathrm{~min}$. The supernatant was quantified for BCA protein concentration using the BCA protein assay kit (Pierce Corp.), as mentioned above. In brief, protein solutions were mixed with appropriate volumes of load buffers and boiled at $100^{\circ} \mathrm{C}$ for $5 \mathrm{~min}$. The protein sample was separated by sodium dodecyl sulfonate-polyacrylamide gel electrophoresis and transferred to a polyvinylidene difluoride membrane by transmembrane reaction. The membrane was blocked by $5 \%$ skimmed milk powder in TBST buffer (Tris-buffered saline and Tween 20) for $1 \mathrm{~h}$, followed by $4^{\circ} \mathrm{C}$ overnight incubation using primary antibody. After washing with TBST three times, the sample was incubated with secondary antibody conjugated with horseradish peroxidase at room temperature for $1 \mathrm{~h}$. After washing with TBST three more times, the membrane was exposed on an X-ray film using the chromogenic substrate (Pierce Corp.). The relative expression level of protein was quantified using the Image $\mathrm{J}$ software (NIH, USA). All antibodies used in this study, including those against cleaved caspase-3, p-eIF $2 \alpha$, eIF $2 \alpha$, cytochrome c, and B-cell lymphoma-extra large (Bcl-xL), were commercially purchased (Santa Cruz, US). Actin was included as the internal reference protein for the Western blot.

\section{Statistical analysis}

SPSS v11.5 (IBM Corp., USA) was used to analyze all collected data. All results are reported as means $\pm \mathrm{SE}$. The comparison among multiple groups was accomplished by analysis of variance. Statistical significance was defined as $\mathrm{P}<0.05$.

\section{RESULTS}

\section{Pretreatment with salubrinal can protect cardiomyocytes against doxorubicin- induced cell apoptosis}

We first determined whether salubrinal had any side effects on neonatal rat cardiomyocytes by measuring the level of lactate dehydrogenase. The results showed no significant change in the level of lactate dehydrogenase in neonatal rat cardiomyocytes, even after pretreatment with $40 \mu \mathrm{M}$ salubrinal (Figure 1A). A further TUNEL assay revealed that salubrinal protects against doxorubicin-induced cardiomyocyte apoptosis: the number of TUNEL-positive cells after pretreatment with 20 or $40 \mu \mathrm{M}$ salubrinal was significantly lower than that observed without salubrinal treatment (Figure 1B; P $<0.05$ in both cases). A decrease in the TUNEL-positive cell number also occurred in cardiomyocytes pretreated with $10 \mu \mathrm{M}$ salubrinal, although the difference was not statistically significant (Figure 1B; P > 0.05). 

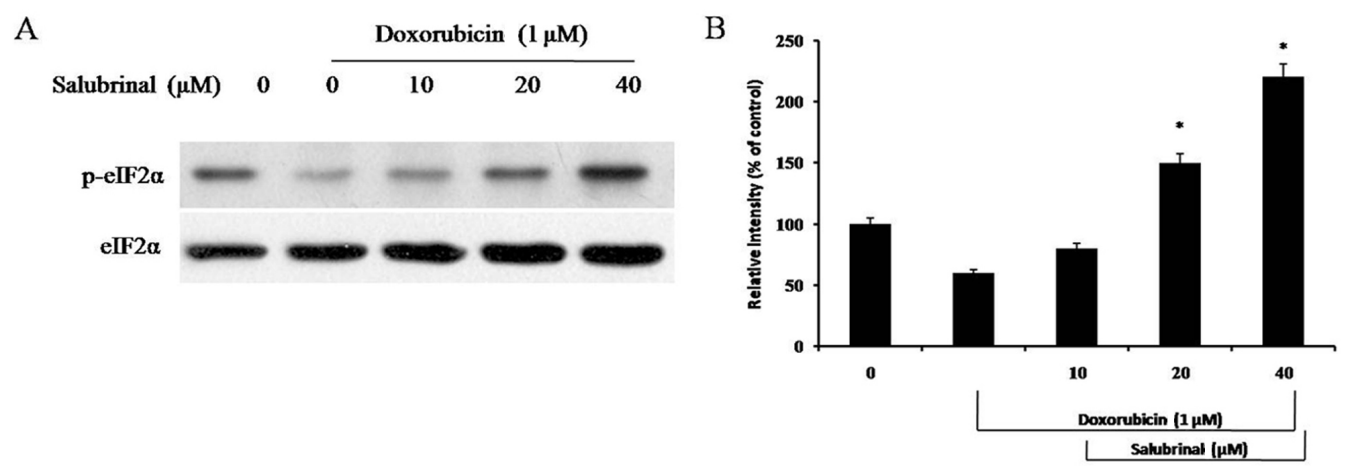

Figure 1. Doxorubicin-induced cardiomyocyte apoptosis following salubrinal pretreatment. A. Effect of salubrinal pretreatment on lactase dehydrogenase level; B. terminal deoxynucleotidyl transferase (TdT)-mediated deoxyuridine triphosphate nick end-labeling (TUNEL)-positive cardiomyocytes undergoing salubrinal pretreatment and doxorubicin-induced apoptosis. $* \mathrm{P}<0.05$.

\section{Effect of salubrinal pretreatment on cardiomyocyte apoptosis-related protein levels}

The western blot showed that cleaved caspase-3 and cytochrome c, which are both apoptosis-related proteins, had decreasing expression levels after pretreatment with increasing concentrations of salubrinal (Figure 2A and B). The cells that had undergone salubrinal pretreatment had significantly lower protein levels compared with those without salubrinal (P $<0.05$ in both the 20 and $40 \mu \mathrm{M}$ salubrinal groups). However, the level of apoptosis-related protein Bcl-xL increased significantly with increasing concentrations of salubrinal compared with those cells without salubrinal pretreatment $(\mathrm{P}<0.05$ in both 20 and $40 \mu \mathrm{M}$ salubrinal groups; Figure 2C).
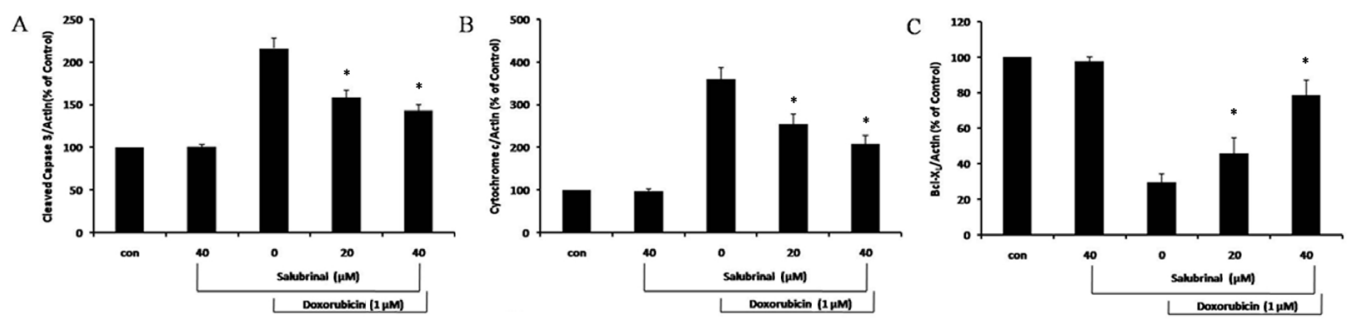

Figure 2. Analysis of doxorubicin-induced cardiomyocyte apoptosis following salubrinal pretreatment. A. Relative change of cleaved caspase-3 protein expression level; B. relative change of cytochrome c protein expression level; C. relative change of B-cell lymphoma-extra large (Bcl-xL) protein expression level. $* \mathrm{P}<0.05$.

\section{Effect of salubrinal pretreatment on reactive oxygen species level and NAPDH oxidase activity}

To further evaluate the function of salubrinal in doxorubicin-induced apoptosis in neonatal rat cardiomyocytes, the level of reactive oxygen species and the activity of NADPH oxidase were further quantified. The results showed a significantly lower level of reactive oxygen species in neonatal rat cardiomyocytes after pretreatment with 20 or $40 \mu \mathrm{M}$ salubri- 
nal compared with cells that had not received pretreatment and had undergone doxorubicin incubation alone ( $\mathrm{P}<0.05$ in both cases; Figure 3A). The cytosolic peroxide concentration was also measured and the results showed a significantly lower peroxide level in neonatal cardiomyocytes following salubrinal pretreatment $(20$ or $40 \mu \mathrm{M})$ than in those cells that had received doxorubicin treatment only $(\mathrm{P}<0.05$ in both cases; Figure $3 \mathrm{~B})$. Furthermore, the NADPH oxidase activity of the intracellular microsomes was significantly decreased following pretreatment with 20 or $40 \mu \mathrm{M}$ salubrinal $(\mathrm{P}<0.05$ in both cases compared with those cells receiving doxorubicin but no salubrinal; Figure $3 \mathrm{C}$ ).
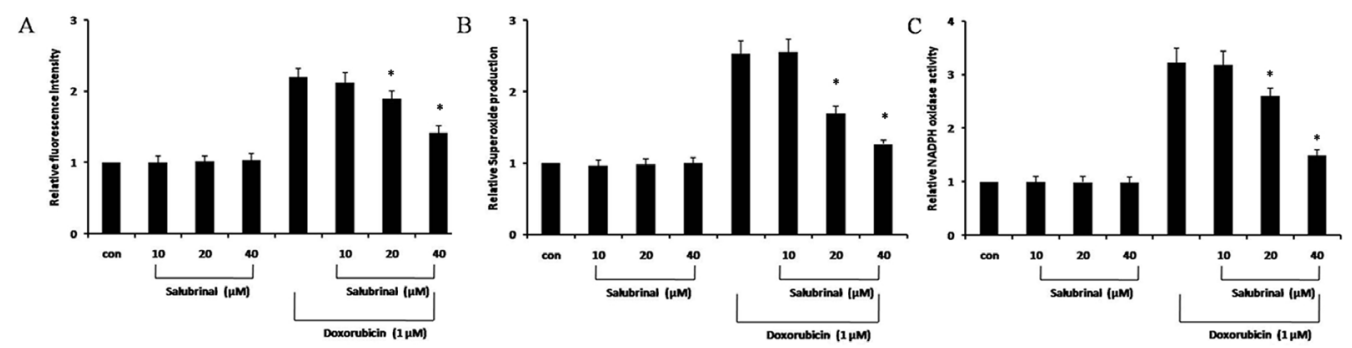

Figure 3. Measurement of reactive oxygen species production (A); superoxide generation (B); and nicotinamide adenine dinucleotide phosphate (NADPH) oxidase activity $(\mathbf{C}) .{ }^{*} \mathrm{P}<0.05$.

\section{Salubrinal protected neonatal rat cardiomyocytes by inhibiting eIF $2 \alpha$ dephos- phorylation}

To study the molecular mechanism by which salubrinal protects neonatal rat cardiomyocytes, we measured the phosphorylation level of eIF $2 \alpha$. The results showed that following pretreatment with 20 or $40 \mu \mathrm{M}$ salubrinal, the level of dephosphorylation in eIF $2 \alpha$ in the cardiomyocytes was significantly lower than that in the control group without salubrinal $(\mathrm{P}<$ 0.05 in both cases; Figure 4A and B). Cells pretreated with $10 \mu \mathrm{M}$ salubrinal showed a minor decrease in eIF $2 \alpha$ dephosphorylation, although at a statistically insignificant level $(\mathrm{P}>0.05$; Figure $4 \mathrm{~A}$ and $\mathrm{B}$ ).
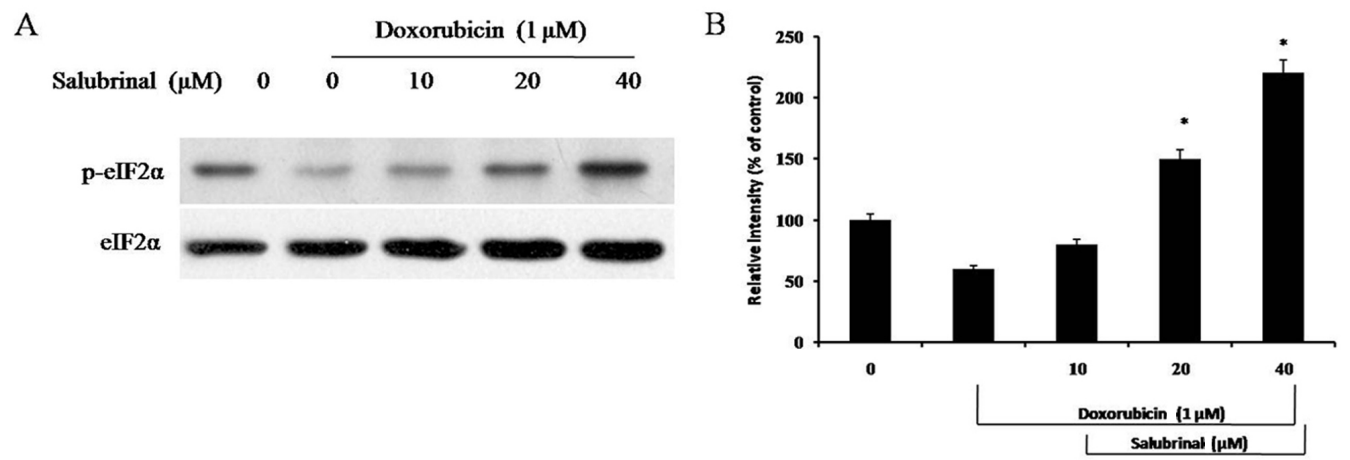

Figure 4. Protection of cardiomyocytes against doxorubicin-induced cell apoptosis by salubrinal. A. Level of phosphorylation of eukaryotic translation initiation factor $2 \alpha$ (eIF2 $\alpha$ ); B. relative intensity produced by phosphorylated eIF $2 \alpha$ (p-eIF $2 \alpha)$. $* \mathrm{P}<0.05$. 


\section{DISCUSSION}

This study aimed to determine whether salubrinal pretreatment protects against doxorubicin-induced cell apoptosis in primary-cultured neonatal rat cardiomyocytes. Our results showed a significantly reduced number of doxorubicin-induced apoptotic cardiomyocytes following salubrinal pretreatment. Furthermore, the production of reactive oxygen species and the activity of NADPH oxidase were significantly reduced following salubrinal pretreatment. Regarding the functional mechanism, we discovered a significantly decreased dephosphorylation level in eIF $2 \alpha$ in neonatal rat cardiomyocytes following salubrinal pretreatment. These data all suggest that salubrinal protects cardiomyocytes against doxorubicin-induced cell apoptosis.

As a selective inhibitor of eIF2 $\alpha$, salubrinal has been reported to protect rat neural cell line PC-12 against tunicamycin-induced endoplasmic reticulum stress response (Paschen, 2003). A further study determined the potency of salubrinal in ameliorating neurotoxicity produced by cyclosporine-induced endoplasmic reticulum stress response in rats (Gasparetto et al., 2004). Furthermore, salubrinal has been used in the treatment of patients with urinary disease (Katsoulieris et al., 2009) and diabetes (Cnop et al., 2007). A more recent study has shown that salubrinal can effectively inhibit cardiomyocyte apoptosis induced by tunicamycin or hypoxia, possibly by its regulation of the pERK-eIF2 $\alpha$ signaling pathway (Liu et al., 2012). These studies support the hypothesis of the protective role of salubrinal against doxorubicininduced cardiomyocyte apoptosis, which was the focus of our study.

Cardiomyocytes that have been treated with doxorubicin for $16 \mathrm{~h}$ show the typical characteristics of cell apoptosis, such as staggered DNA bands under gel electrophoresis (Negoro et al., 2001). In this study, we utilized a similar in vitro model to that reported by Negoro et al. (2001) to determine if pretreatment with salubrinal of different concentrations can protect cardiomyocytes from doxorubicin-induced cell apoptosis. Furthermore, reactive oxygen species play an important role in doxorubicin-induced cardiomyocyte apoptosis. Consistent with previous reports (Park et al., 2011), our study demonstrated the inhibitory function of salubrinal in the production of reactive oxygen species. NAPDH oxidase, which is one of the enzymes involved in the production of superoxide, is mainly expressed in blood vessels, and participates in multiple pathogeneses including cardiac fibrosis (Yisireyili et al., 2013), malfunctions in endothelial cells (Galán et al., 2014), and atherosclerosis (Gray et al., 2013). Additionally, the genetic polymorphism of three subunits of NADPH oxidase is related to doxorubicin-induced cardiac toxicity. Our research found that salubrinal pretreatment could significantly reduce NADPH oxidase activity, which, at high levels, along with the production of reactive oxygen species, is believed to be the major cause of doxorubicin-induced cardiac pathology (Deng et al., 2007).

Because some reports have suggested that salubrinal exerts its protective functions mainly by its regulatory effects on eIF2 $\alpha$ (Boyce et al., 2005; Wiseman and Balch, 2005), we also measured the expression pattern of eIF $2 \alpha$, which showed a lower dephosphorylation level after salubrinal pretreatment.

In summary, this study suggests that salubrinal pretreatment can effectively protect cardiomyocytes against doxorubicin-induced apoptosis by inhibiting eIF2 $\alpha$ dephosphorylation. However, some limitations exist as no in vivo study has been performed to substantiate this working model. Further studies are therefore required to determine the potency of salubrinal in ameliorating cardiac toxicity in the body. 


\title{
Conflicts of interest
}

The authors declare no conflict of interest.

\section{ACKNOWLEDGMENTS}

\author{
We thank the anonymous reviewers for reviewing this manuscript.
}

\section{REFERENCES}

Boyce M, Bryant KF, Jousse C, Long K, et al. (2005). A selective inhibitor of eIF2alpha dephosphorylation protects cells from ER stress. Science 307: 935-939.

Chen YL, Liu JC, Loh SH, Chen CH, et al. (2008). Involvement of reactive oxygen species in urotensin II-induced proliferation of cardiac fibroblasts. Eur. J. Pharmacol. 593: 24-29.

Chua CC, Liu X, Gao J, Hamdy RC, et al. (2006). Multiple actions of pifithrin-alpha on doxorubicin-induced apoptosis in rat myoblastic H9c2 cells. Am. J. Physiol. Heart Circ. Physiol. 290: H2606-H2613.

Cnop M, Ladriere L, Hekerman P, Ortis F, et al. (2007). Selective inhibition of eukaryotic translation initiation factor 2 alpha dephosphorylation potentiates fatty acid-induced endoplasmic reticulum stress and causes pancreatic beta-cell dysfunction and apoptosis. J. Biol. Chem. 282: 3989-3997.

Dalal S, Foster CR, Das BC, Singh M, et al. (2012). B-adrenergic receptor stimulation induces endoplasmic reticulum stress in adult cardiac myocytes: role in apoptosis. Mol. Cell Biochem. 364: 59-70.

Deng S, Kruger A, Kleschyov AL, Kalinowski L, et al. (2007). Gp91phox-containing NAD(P)H oxidase increases superoxide formation by doxorubicin and NADPH. Free Radic. Biol. Med. 42: 466-473.

Eruslanov E and Kusmartsev S (2010). Identification of ROS using oxidized DCFDA and flow-cytometry. Methods Mol. Biol. 594: 57-72.

Galán M, Kassan M, Kadowitz PJ, Trebak M, et al. (2014). Mechanism of endoplasmic reticulum stress-induced vascular endothelial dysfunction. Biochim. Biophys. Acta. 1843: 1063-1075.

Gasparetto M, Gentry T, Sebti S, O'Bryan E, et al. (2004). Identification of compounds that enhance the anti-lymphoma activity of rituximab using flow cytometric high-content screening. J. Immunol. Methods 292: 59-71.

Gray SP, Di Marco E, Okabe J, Szyndralewiez C, et al. (2013). NADPH oxidase 1 plays a key role in diabetes mellitusaccelerated atherosclerosis. Circulation 127: 1888-1902.

Juan SH, Chen CH, Hsu YH, Hou CC, et al. (2007). Tetramethylpyrazine protects rat renal tubular cell apoptosis induced by gentamicin. Nephrol. Dial. Transplant. 22: 732-739.

Katsoulieris E, Mabley JG, Samai M, Green IC, et al. (2009). Alpha-linolenic acid protects renal cells against palmitic acid lipotoxicity via inhibition of endoplasmic reticulum stress. Eur. J. Pharmacol. 623: 107-112.

Lipshultz SE and Adams MJ (2010). Cardiotoxicity after childhood cancer: beginning with the end in mind. J. Clin. Oncol. 28: 1276-1281.

Lipshultz SE, Alvarez JA and Scully RE (2008). Anthracycline associated cardiotoxicity in survivors of childhood cancer. Heart 94: 525-533.

Liu CL, Li X, Hu GL, Li RJ, et al. (2012). Salubrinal protects against tunicamycin and hypoxia induced cardiomyocyte apoptosis via the PERK-eIF2 $\alpha$ signaling pathway. J. Geriatr. Cardiol. 9: 258-268.

Negoro S, Oh H, Tone E, Kunisada K, et al. (2001). Glycoprotein 130 regulates cardiac myocyte survival in doxorubicininduced apoptosis through phosphatidylinositol 3-kinase/Akt phosphorylation and Bcl-xL/caspase-3 interaction. Circulation 103: 555-561.

Oeffinger KC, Mertens AC, Sklar CA, Kawashima T, et al. (2006). Chronic health conditions in adult survivors of childhood cancer. N. Engl. J. Med. 355: 1572-1582.

Park GB, Kim YS, Lee HK, Song H, et al. (2011). Reactive oxygen species and p38 MAPK regulate Bax translocation and calcium redistribution in salubrinal-induced apoptosis of EBV-transformed B cells. Cancer Lett. 313: 235-248.

Paschen W (2003). Endoplasmic reticulum: a primary target in various acute disorders and degenerative diseases of the brain. Cell Calcium 34: 365-383.

Silverman LB, Stevenson KE, O’Brien JE, Asselin BL, et al. (2010). Long-term results of Dana-Farber Cancer Institute ALL Consortium protocols for children with newly diagnosed acute lymphoblastic leukemia (1985-2000). Leukemia 24: $320-334$. 
Tarver T (2012). Cancer Facts \& Figures 2012. American Cancer Society (ACS) American Cancer Society, Atlanta. Available at [http://www.cancer.org/research/cancerfactsfigures/cancerfactsfigures/cancer-facts-figures-2012]. Accessed March 3, 2015. J. Cons. Health Internet. 16: 366-367.

Tukenova M, Guibout C, Oberlin O, Doyon F, et al. (2010). Role of cancer treatment in long-term overall and cardiovascular mortality after childhood cancer. J. Clin. Oncol. 28: 1308-1315.

Wang XY, Yang CT, Zheng DD, Mo LQ, et al. (2012). Hydrogen sulfide protects H9c2 cells against doxorubicin-induced cardiotoxicity through inhibition of endoplasmic reticulum stress. Mol. Cell Biochem. 363: 419-426.

Wiseman RL and Balch WE (2005). A new pharmacology - drugging stressed folding pathways. Trends Mol. Med. 11: 347-350.

Wung BS, Cheng JJ, Hsieh HJ, Shyy YJ, et al. (1997). Cyclic strain-induced monocyte chemotactic protein-1 gene expression in endothelial cells involves reactive oxygen species activation of activator protein 1. Circ. Res. 81: 1-7.

Yisireyili M, Shimizu H, Saito S, Enomoto A, et al. (2013). Indoxyl sulfate promotes cardiac fibrosis with enhanced oxidative stress in hypertensive rats. Life Sci. 92: 1180-1185. 